\title{
Análisis de los estándares de calidad y su incidencia en el desempeño docente de la Educación General Básica y Bachillerato de la Unidad Educativa Catalina Cadena del Cantón el Triunfo
}

\section{Analysis of the quality standards and their impact on the teaching performance of the General Basic and Baccalaureate Education of the Catalina Cadena of Canton el Triunfo Educational Unit}

\author{
MSc, Adriana Olmedo Valencia \\ aolmedo@gmail.com \\ PhD. Milton Maridueña ${ }^{2}$ \\ mmaridueña@gmail.com
}

Recibido: 1/12/2018; Aceptado: 1/02/2019

\begin{abstract}
RESUMEN
Esta investigación trata sobre los estándares de calidad y su incidencia en el desempeño docente de la educación general básica y bachillerato. Como muestra se escogió la Unidad Educativa Catalina Cadena Miranda, ubicado en el Cantón El Triunfo. El objetivo de este estudio es el de analizar y ampliar el conocimiento de los estándares de calidad en el campo educativo para fortalecer el proceso de enseñanza- aprendizaje de los docentes y que a su vez los estudiantes puedan alcanzar los aprendizajes deseados y desarrollen destrezas para su formación como un ciudadano útil a la sociedad. Se consideró el distributivo docente del Dpto. de Talento Humano de la Dirección Distrital 09D16 el Triunfo-Gral. Antonio Elizalde; para el marco teórico se tomó documentos de apoyo del Ministerio de Educación, libros y artículos científicos relacionados al campo educativo que enfocan la calidad y el desempeño docente, el diseño de la investigación tomo un enfoque cualitativo que incluye los métodos de análisis y síntesis.
\end{abstract}

\footnotetext{
1 Estudiante de Maestría UTEG. Ecuador

2 Docente Posgrado UTEG. Ecuador
} 
Se aplicó el método analítico-sintético e inductivo-deductivo y un análisis detallado de los estándares específicos que requiere el docente para un desarrollo de desempeño en el aula. El método histórico-lógico accedió a formar los antecedentes de la evaluación del desempeño. Se complementó con una guía de observación, para realizar una investigación de campo correspondiente a los indicadores de desempeño que están vinculadas a los estándares de calidad profesional en los niveles de básica superior y bachillerato. El análisis permitió determinar que los docentes desconocen de los estándares de calidad para el desempeño profesional y que es necesario fomentar en el aula una enseñanza que permita que todos los educandos ecuatorianos alcancen los perfiles de egreso por el currículo nacional para la educación.

Palabras Clave: dimensión, aprendizaje, desempeño docente, calidad educativa

\section{ABSTRACT}

This research deals with quality standards and their incidence in the teaching performance of general basic education and baccalaureate. The Unidad Educativa Catalina Miranda, located in El Triunfo, was chosen for analysis. The objective of this study is to analyze and expand knowledge of quality standards in the field of education and to strengthen the teaching-learning process of teachers, and in turn students can achieve the desired learning and develop skills for their training as a useful citizen to society. The teachers' list District Directorate 09D16 El TriunfoGeneral Antonio Elizalde, from the Human Resources Department was taken into account. For the theoretical framework, supporting documents of the Ministry of Education, books and scientific articles related to the educational field focusing on quality and teaching performance were used as references. Design is based on a qualitative approach that includes the methods of analysis and synthesis. The analytical-synthetic and inductive-deductive method was used and as well as an evaluation of the teacher's professional performance in the classroom. The historicallogical method helped in analyzing the performance's evaluation. This research was complemented with the use of the observation guide, therefore facilitating an evaluation of performance indicators that are linked to professional quality standards. Results demonstrated that teachers are unaware of quality standards for professional performance and that it is necessary to promote in the classroom a teaching that allows all Ecuadorian students reach the exit profiles for the national curriculum for education.

Keywords: dimension, learning, teaching performance, educational quality

\section{Introducción}

Perfeccionar la calidad de la educación es, en la actualidad, el importante reto de los países del mundo. La colectividad moderna cada vez más globalizada, se identifica por una nueva y sorprendente revolución científico-técnica, lo que aplica a las políticas educativas y a la orientación escolar de altos requerimientos, en aras de progresar y/o perfeccionar la calidad del transcurso de educación desde una perspectiva más integradora.

Aunque se pueda estar en principio de acuerdo en que es significativo promover la calidad educativa, el estimado de la expresión "calidad educativa" es debatido porque persistentemente tendrá un vínculo histórico ya que puede desarrollarse en el tiempo y figurará un cierto ideal o anhelo de la sociedad en su conjunto o de grupos por sí 


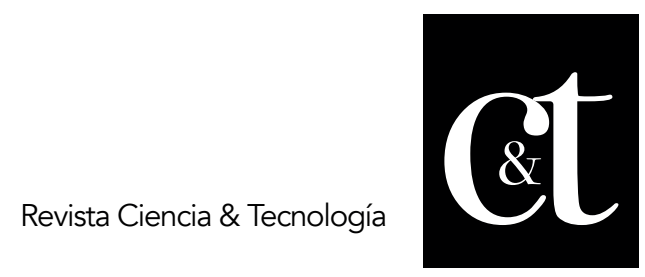

No. 22, 30 de abril de 2019

ISSN impreso: 1390 - 6321

ISSN online: 2661 - 6734

mismo. La diversidad de composturas existentes siempre le va a determinar a lo que cada individuo o grupo de personas discurre que debe ser el fin o intención principal de la educación como actividad humana. Consecuentemente, no se trata de un concepto neutro, sino que tiene una fuerte carga social, económica, cultural y política.

La intención que especificamos para la educación es concluyente para dar sentido a la expresión "calidad educativa". Varios creen equivocadamente que existe una aprobación sobre cuáles deben ser los desenlaces de la tarea educativa, cuando en realidad esto depende de la condición como las personas crean la sociedad ideal, cuyo éxito debería aportar el sistema educativo como componente de socialización y de creación de capacidades y emprendimiento para evolucionar y mejorar la sociedad.

La calidad de educación está vinculada con la rendición de cuentas que en los últimos años han sido incluidos en el marco legal de los países latinoamericanos como es el caso del Estado del Ecuador de acuerdo a lo que consta en la Constitución de la República del 2008 que el Artículo... dice:

"Ante la alta responsabilidad de velar por la calidad de educación que se imparta en las aulas de los niveles de Educación que funcionan en el Ecuador con el propósito de formar ciudadanos que al final de cuenta tiene que ver con el nivel de vida que aspira la sociedad. Los estándares de calidad educativos son representaciones de los resultados esperados correspondientes a los diferentes actores e instituciones del sistema educativo".

Según Urbay Rodríguez (2006) en el empeño por perfeccionar el sistema educativo y mejorar la calidad del impacto de éste, se ha determinado como elemento importante el desempeño profesional del docente que contribuirá al cambio en el proceso educacional. El éxito de cualquier sistema educativo dependerá en gran medida del nivel de desarrollo profesional alcanzado por los docentes lo que repercutirá en la calidad de los resultados del aprendizaje.

Un docente con eficacia y eficiencia es aquel que proporciona circunstancias de enseñanzas a todos los estudiantes y ayuda, mediante su formación, a edificar la sociedad que deseamos para nuestro país.

La intención de los estándares de desempeño docente es promover en el salón de clases, una educación que permita que todos los estudiantes ecuatorianos logren los perfiles señalados por el currículo nacional para la Educación General Básica y para el Bachillerato.

Conjuntamente, los estándares de desempeño profesional docente constituyen las particularidades y desempeños generales y básicos que corresponden realizar los docentes para desenvolver un transcurso de enseñanza-aprendizaje de calidad.

Es inevitable precisar que el docente ya no es únicamente el servidor de un EstadoNación que instruye a unos y deja fuera a otros. Su compromiso se dirige a una sociedad crecientemente heterogénea y plural, que demanda para todos sus jóvenes un ejemplo de educación que los prepare, no sólo para su participación ciudadana, sino también para su participación productiva.

El liderazgo de un docente tiene impacto significativo en el desempeño académico en 


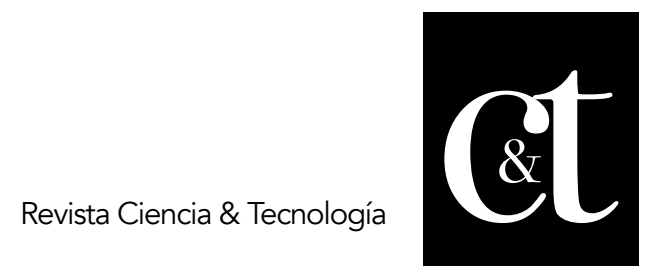

No. 22, 30 de abril de 2019

ISSN impreso: 1390 - 6321

ISSN online: 2661 - 6734

el aula. Por esta razón, un liderazgo efectivo y concordante inspira la auto capacitación, búsqueda y adopción de nuevas prácticas educativas más efectivas, a nuevas formas de equipos de trabajo y de aprendizaje organizacional, con el objetivo de lograr la vinculación y participación de sus representantes legales, docentes, estudiantes de niveles educativos básica superior y bachillerato y comunidad en el procesos educativo, obviamente se podrá ver reflejado y constituirá de manera significativa que la Institución Educativa cumpla con sus objetivos de una manera más eficiente y positiva.

La atención a cabalidad de estándares de desempeño en la formación docente, no puede omitir las situaciones laborales de los docentes, tanto para quienes están en acción como para quienes se constituyen y miran con atención dicho escenario. Se requiere ajustar las oportunidades de formación en servicio; crear una concordancia entre un desempeño efectivo y de calidad, con trayectos de una carrera docente que no tenga como único fin un cargo en la administración. Esto es, un método efectivo de valoración formativa del desempeño. Una política integral debe hacer viable la formulación de fines de desempeño que marquen inmediatos a lo largo de la carrera profesional, que consiguen describirse como juicios de desempeño y que indiquen a los objetivos educacionales en su conjunto y, más concretamente, a las instancias de la enseñanza de los currículos.

La realización de esta investigación se justifica por la necesidad de calidad y eficiencia que deben tener los docentes en su desempeño profesional en el aula de clases.

\section{Desarrollo}

El desempeño de los maestros es un factor imprescindible para que la calidad de aprendizajes en el aula sea de buena calidad. Un docente que tiene su formación en las Facultades de Educación, es decir que su perfil profesional sea acorde con la asignatura que desarrolla en el aula, además que esté constantemente actualizando sus conocimientos en Seminarios, talleres y congresos educativos es un buen referente de un proceso de enseñanza acorde con los estándares de calidad de la educación requeridos.

Desde el 2006 en el que este país, en concordancia con el Plan Decenal (2006) ya adoptó la cultura evaluativa al igual que otros países de Latinoamérica. Es necesario que los docentes tengan un dominio conceptual acorde con los estándares de calidad y evidencie una alta comprensión de los indicadores relacionados con su desempeño, esta condición también coadyuvará para que contribuya a un desempeño de calidad.

En el Documento emitido por el Ministerio de Educación en el 2011 denominado Estándares de Desempeño Profesional Docente: Propuesta para la Discusión ciudadana se manifiesta que para establecer qué es una educación de calidad, se necesita primero identificar "qué tipo de sociedad queremos tener, pues un sistema educativo será de calidad en la medida que contribuya a la consecución de esa meta" (p. 4).

Cuando se toma como punto de partida a la sociedad formada por seres humanos que son quienes en realidad construyen un país y depende de ello su formación intelectual, sus valores y el respeto por las costumbres el Ministerio de Educación fundamenta muy bien este criterio. Acertado el consultar con la ciudadanía 


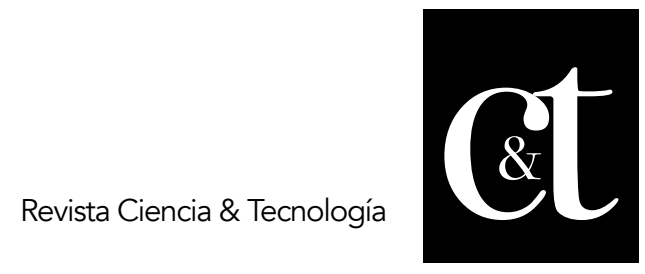

No. 22, 30 de abril de 2019

ISSN impreso: 1390 - 6321

ISSN online: 2661 - 6734

principalmente a los docentes del Ecuador en el 2011 para que puedan empaparse en lo concerniente a los Estándares de calidad, más aún cuando en el documento oficial se exponen los Indicadores referente a su desempeño.

La Constitución del Ecuador en el artículo 26 contempla "la educación es un derecho de las personas a lo largo de su vida y un deber ineludible e inexcusable del Estado" además en el artículo 27 consta que "la educación debe ser de calidad". Por lo tanto es una gran responsabilidad para todos los actores del Ministerio de Educación aportar para que la educación en todos los establecimientos del país se desarrollen bajo los parámetros de calidad. Es menester analizar en que consiste la calidad educativa.

Laies (2011) relaciona la calidad con el enfoque educativo a propósito de la eficacia, el cumplimiento de los objetivos propuestos, los recursos disponibles y el contexto con respecto a la pertinencia y al mundo en que viven los estudiantes. Por otra parte, Bürgi \& Peralta en el (2011) analizaron con profundidad los factores que inciden en la calidad educativa y encuentran que guardan relación con la orientación al logro, al alto rendimiento y la eficacia. En Bernal, Martínez, Parra y Jiménez (2015) los autores mucho acierto al decir que la calidad educativa se puede apreciar desde cuatro subconjuntos:

1. Ethos: formas de comprender lo social y las perspectivas paradigmáticas de la educación

2. Procesos de enseñanza y aprendizaje

3. La organización escolar, es decir, todos los aspectos del ámbito educativo

4. Factores externos, condiciones de vida

Estos autores enfatizan aspectos claves referentes a calidad educativa que es lo que realmente influye en el tipo de ciudadano que se debe formar desde la gestión educativa comenzando desde las aulas. Cabe también manifestar que la gestión que se realice en los establecimientos educativos debe incluirse a la familia para que este factor sea un aporte a la formación del ser humano y no un limitante. La condición de vida es un factor externo que índice grandemente en la formación del niño y el adolescente que incluye Educación Inicial, Básica y Bachillerato.

La UNESCO, un organismo vinculado con la educación que tiene a cargo la coordinación de los sistemas educativos en los países de América Latina y el Caribe, mediante la Red de los Sistemas de Medición y Evaluación de la calidad de Educación desde la década del 80 se ha establecido un laboratorio que se encarga del análisis de variables que inciden en la forma de conseguir mejores logros en los aprendizajes en la Educación Básica. Uno de los referentes es la estructura de estándares de calidad.

Según Casassus (2007), hay conceptos fundamentales y la calidad de la educación expone que, sólo a partir de los años 90 se introdujo la temática de los estándares de educación, que en los sistemas educativos recientemente a través de los Ministerios de Educación se ha tomado conciencia de la responsabilidad en temas del fracaso escolar y los factores vinculantes, que una de las razones de no superar la sensación de fraude es la ausencia de estándares de calidad que indiquen cuál es el 


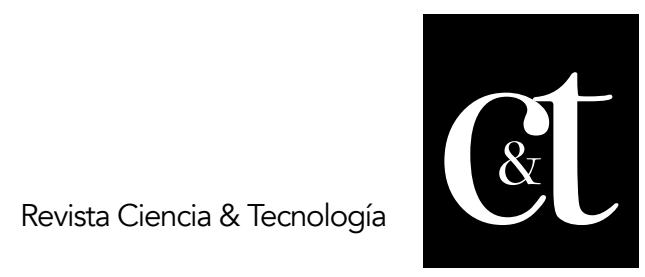

compromiso del Sistema Educativo con la sociedad (UTPL, 2013).

En el documento antes indicado se incorpora el ¿Por qué desarrollar estándares de Educación?, entre las opiniones vertidas se afirma que al visualizar falencias en los estudiantes que logran terminar sus estudios cuando presentan dificultades de redacción, dificultades de expresión y pocas destrezas en cuanto al cálculo. Esta es una de las razones de peso que lleva a dar una respuesta de la necesidad de formular estándares de calidad.

Cabe indicar que el documento que se describe especifica en qué consiste cuatro conceptos que deben quedar muy en claro para vincularlos con estándares de calidad estos son: las aptitudes, capacidades, competencias y destrezas. Al visualizar los estándares relacionados al aprendizaje de los estudiantes se puede evaluar lo que el sistema educativo debe ser capaz de generar para satisfacción o insatisfacción del usuario.

En la publicación del 2001 del Ministerio de Educación de Chile logró realizar consenso con las instituciones de formación docente, el Colegio de Profesores para establecer los Estándares de Desempeño para la formación de Docentes, lo importante de este gran paso es que todas las partes estuvieron orientadas a aportar al mejoramiento de la calidad de desempeño docente. En este documento se describe la misión y tareas del docente y se establecen los Estándares de desempeño con base del proceso de enseñanza aprendizaje que se desarrolla en las aulas.

Para complementar este gran aporte para mejorar la calidad educativa en uno de los países de América Latina, se expone parte del documento Estándares de Desempeño Docente, que corresponde a la Universidad Católica Silva Henríquez (2017), en el que se hace conocer mediante Reyes, L. (2017) que los Estándares de Desempeño para la formación Inicial de Docentes, fueron descritos estableciendo una especie de glosario y además se definió los estándares se expresan como criterios que orientan la evaluación del desempeño de un docente en cuatro áreas de acción que se denominan facetas y son las siguientes.

Preparación del acto de enseñar

Es menester manifestar que desde la década del 90 los Estados Latinoamericanos han evidenciado la preocupación respeto a la necesidad de gestionar estrategias relativas para aportar a la calidad educativa que se desarrolla en los diferentes niveles de Educación, constituyéndose en mayor preocupación por trabajar en nivel de Educación Inicial y Básica.

En el 2002 el Ecuador emite un referente curricular de la Educación Inicial, en el 2008 se inicia una capacitación masiva dirigida a docentes del país a través de la cual se socializa la Reforma Curricular con el propósito de que los docentes se actualicen en la nueva forma de llevar adelante el currículo en los Establecimientos Educativos.

El Estado del Ecuador en el 2006 también se une al Plan Decenal de Educación, Programa que se lanzó a través de los medios de comunicación que tiene el propósito de enfatizar puntos a favor de la calidad de educación de los estudiantes ecuatorianos.

En el 2011 El Ministerio de Educación del Ecuador emite como parte la Revolución 


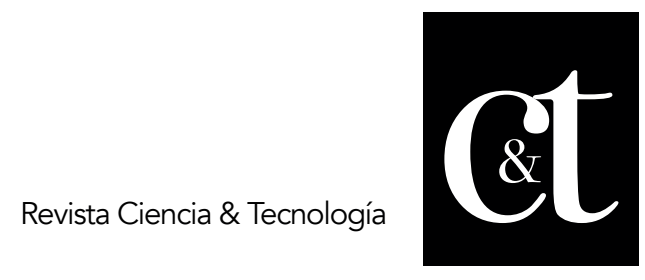

No. 22, 30 de abril de 2019

ISSN impreso: 1390 - 6321

ISSN online: 2661 - 6734

Ciudadana Estándares de Desempeño Profesional Docente, como una Propuesta para la discusión Ciudadana, este Programa estuvo respaldado por la Organización de Estados Iberoamericanos. En este documento, se describe los Estándares de Calidad de Educación; se Incluye la Fundamentación teórica de la propuesta de estándares de desempeño profesional docente y se complementa con el Modelo de estándares de desempeño docente (Méndez y González, 2013).

En el 2012 mediante el Acuerdo Ministerial 0482, el Ministro de Educación, pone a disposición de los docentes los Estándares de Calidad Educativa, Este Acuerdo viene con el anexo que está conformado por Estándares Específicos referentes a Aprendizaje, Gestión Escolar, Desempeño Profesional e Infraestructura. Se describe de forma explícita el significado de calidad educativa; definiciones de Estándares de Calidad Educativa y en el tercer capítulo se describe respecto a los estándares específicos.

En el 2017, el Ministerio de Educación dispone una reforma al documento del 2012 mediante Acuerdo Nro. Mineduc- Mineduc - 2017 -00091, este documento oficial consiste en que se expone una sustitución de los anexos correspondientes a los literales a) y b) del artículo 1 de los Estándares de Gestión Escolar, Desempeño Profesional Docente y Desempeño Profesional Directivo y afirma que serán referentes para la evaluación interna y externa orientada a la medición de la gestión escolar y desempeño docente y directivo. Agrega también que; estos estándares serán utilizados por el Instituto de Evaluación Educativa INEVAL o por cualquiera otra institución que realice evaluación externa (López, 2000).

Ortiz, Fabara, Villagómez e Hidalgo (2017) afirmaron que ESTRADO (Red Latinoamericana de Estudios sobre Trabajo Docente) se constituyó en el Ecuador en el 2012.

En el 2017 en el Acuerdo Ministerial Nro. Mineduc-Mineduc-2017-00091- el Ministro indicó que:

Que, la Constitución de la República del Ecuador en sus artículos 26 y 27 establece que la educación es un derecho de las personas y un deber ineludible e inexcusable del Estado, que constituye un área prioritaria de la política pública, garantía de la igualdad e inclusión social y condición indispensable para el buen vivir; y que la educación se centrará en el ser humano y garantizará su desarrollo en el marco del respeto de los derechos humanos, e impulsará la justicia, la solidaridad y la paz.

El aprendizaje efectivo deberá incluir características escolares, docentes lideres educativos, currículo, perfil del docente, infraestructura y características extraescolares estas son: los estudiantes, padres de familia, el nivel socioeconómico y cultural, esto determinara las oportunidades de aprendizajes y garantizara mejores efectos. Toranzos (2016): "En la búsqueda de estándares de calidad", Organización de Estados Iberoamericanos-OEI, pp. (1-5) manifiesta lo siguiente: Una escuela de calidad, un sistema educativo de calidad es aquel que garantiza oportunidades de aprendizaje equivalentes para todos los alumnos, es decir aquel que se preocupa por generar una conjunción favorable entre los factores externos e internos a la escuela para el desarrollo de resultados exitosos en términos de aprendizaje. 
Como se ha indicado en los párrafos anteriores es importante que los docentes apliquen en el aula los estándares de calidad para proveer oportunidades de aprendizaje a todos los estudiantes y a su vez contribuir al desarrollo de destrezas para su vida profesional y social. Para ello los objetivos de los estándares según Ministerio de Educación (2016):

- están planteados dentro del marco del Buen Vivir;

- respetan las diversidades culturales de los pueblos, las etnias y las nacionalidades;

- aseguran la aplicación de procesos y prácticas institucionales inclusivas;

- contribuyen al mejoramiento de la calidad de los procesos de enseñanzaaprendizaje;

- favorecen el desarrollo profesional de todos los actores educativos, y

- vigilan el cumplimiento de los lineamientos y disposiciones establecidos por el Ministerio de Educación.

Los estándares de desempeño profesional docente, se dividen en cuatro dimensiones: Dimensión A: Dominio disciplinar y curricular, este se refiere a que el docente debe conocer, comprender y dominar el área que va a enseñar, por tal motivo es importante que el docente al dictar sus clases este acorde al perfil profesional que posee. Una discusión más ampliada de este tema la realizan Méndez y González (2013). En algunas ocasiones, estas evaluaciones de desempeño docente pueden constituirse en fuente de disputa pero también de oportunidades, según Román y Murillo (2008).

Además, el docente debe conocer el currículo nacional que le ayudará a comprender los componentes de la estructura curricular, los ejes transversales para aplicarlos en el aula. Dimensión B: Gestión de aprendizaje, aquí el docente debe planificar sus clases para el proceso de enseñanza a los estudiantes e incluye en sus planificaciones actividades de aprendizaje y procesos evaluativos de acuerdo a las necesidades de sus estudiantes para que ellos apliquen sus conocimientos y relacionen sus propios procesos de aprendizaje. Dimensión C: Desarrollo Profesional este también es uno de los más importantes porque el docente es el que auto capacita respecto a avances e investigaciones en enseñanza del área que imparte a sus alumnos, reflexiona antes, durante y después de su labor sobre el impacto que causó a sus alumnos.

Dimensión D: el docente promueve valores, tiene altas expectativas y se compromete con el desarrollo de la comunidad, además estimula el acceso y permanencia en el proceso educativo de los estudiantes dentro del sistema educativo.

\section{Conclusiones}

Se puede establecer que generalmente el trabajo del docente se limita al aula de clases, por lo cual la competencia del docente en el proceso de enseñanza aprendizaje a los alumnos no es el adecuado, una de las razones es porque el perfil del docente no está acorde con las asignaturas asignadas al momento de impartir sus clases, la mayoría de los docentes no tienen pedagogía para llegar al estudiante, por tal motivo esta investigación surgió del interés de analizar y aportar a los docentes los estándares de calidad del desempeño profesional docente los Estándares de Calidad 


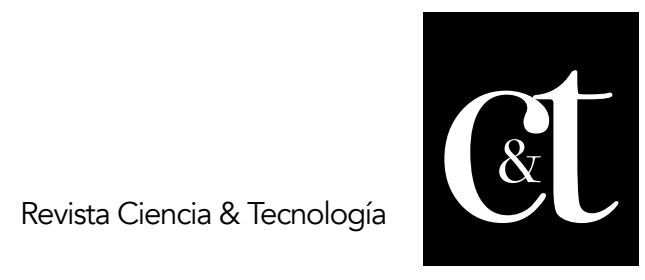

No. 22, 30 de abril de 2019

ISSN impreso: 1390 - 6321

ISSN online: 2661 - 6734

Educativa, Aprendizaje, Gestión Escolar, Desempeño Profesional e Infraestructura. Un docente de calidad es aquel que provee oportunidades de aprendizaje a todos los estudiantes y contribuye, mediante su formación, a construir la sociedad que aspiramos para nuestro país.

Indica que el profesional que imparte sus clases debe de formar al estudiante en su totalidad con el objetivo que pueda ser útil a la sociedad.

Estos estándares permiten que los docentes cumplan con particularidades y ejerza un desempeño de calidad que tenga dominio de la asignatura que imparte, esto ayuda a fortalecer su rol en el aula y no solo se enfoque en las asignaturas, sino que mantenga una buena relación con los alumnos y padres de familia además de ética profesional.

Con el objetivo de fortalecer en el salón de clases estudiantes que alcancen los perfiles de aprendizaje declarados por el Currículo Nacional para la Educación General Básica y Bachillerato.

Como se indicaba anteriormente el docente debe dominar la materia que enseña y obtener epistemología del saber que enseña, didácticas de disciplina que imparte y las teorías e investigaciones que la sustentan.

Además, el docente debe conocer el currículo anterior y posterior al curso que dicta sus clases. El lenguaje debe ser el apropiado cuando imparte la asignatura y cuando está fuera de ella.

Por otra parte, el docente debe procurar no incurrir en stress ni en el llamado burnout (agotamiento) mental, por lo que la didáctica debe ser muy activa, según Rodríguez, Hinojosa y Ramírez (2014).

Al tomar conciencia de los estándares específicos aplicará un desempeño de calidad en el aula al impartir sus clases y servirá para que contribuya de forma sustantiva a la formación del niño y adolescente hasta formarlo como un ciudadano útil a la sociedad.

\section{Referencias bibliográficas}

Bernal, D., Martínez, M., Parra, A. y Jiménez, J. (2015). Investigación documental sobre la calidad de la educación en instituciones educativas del contexto iberoamericano. Revista Entramados-Educación y Sociedad. No.2, pp. 107-124.

Bürgi, J. \& Peralta, M. (2011). El concepto de la calidad educativa en las investigaciones sobre la educación en Chile. Reice. Vol. 9(3), pp. 72-93.

Casassus, J. (2007). La educación de ser emocional. Santiago, Chile: Indigo.

ECUADOR, C. P. (31 de marzo de 2011). Derecho Ecuador.com. Obtenido de Derecho Ecuador.com: https://derechoecuador.com/registro-oficial/2011/03/registrooficial-no-416

Estándares de Calidad Educativa, Aprendizaje, Gestión Escolar, Desempeño Profesional e Infraestructura, disponible en file://C:/Users/Portatil/Desktop/MAESTRIA\%20EN\%20MKT/Marco\%20teóric o\%20conceptual/estandares\%20de\%20calidad_2012.pdf [consulta: 20-03- 
2018].

Laies, G. (2011). Resultado del estudio a dos años del término del programa I de Educación. 2do. Seminario internacional de educación integral: la función directiva E.A.L. pp. 13-44.

López, M. (2000) La Evaluación del Desempeño del Personal Docente. Propuesta de un Modelo de Dirección Estratégica. Tesis con opción al título de Master. IPLAC.

ME, Ecuador. (2014). Ministerio del Ecuador. Recuperado el 05 de mayo de 2014, de http://educacion.gob.ec/tips-de-uso/

ME-Ecuador. (2012). Estándares de Calidad Educativa. Quito.

ME-Ecuador. (2012). Marco Legal Educativo. Quito-

Méndez, Luz y González, Mónica. (2011). Escala de estrategias docentes para aprendizajes significativos: diseño y evaluación de sus propiedades psicométricas. Revista Actualidades Investigativas en Educación, 11(3), 1-39.

Méndez, Luz y González, Mónica. (2013). Evaluación de la Escala de estrategias docentes para aprendizajes significativos (EEDAS). Revista Ciencia UANL, 16(60), 58-77.

Ministerio-Educación (2009). Guía de adecuaciones curriculares. Guatemala: Ministerio de Educación de Guatemala.

Ministerio-Educación, E. (2011). Estándares de desempeño profesional docente. Propuesta para la discusión ciudadana. Quito.

Ministerio-Educación, E. (2012). Estándares de calidad educativa. Quito; Editogran

Ministerio-Educación, E. (2012). Marco Legal Educativo. Quito

Ministerio-Educación (2016). Estándares de Calidad Educativa, Aprendizaje, Gestión Escolar, Desempeño Profesional e Infraestructura. Quito, Ecuador; Mineducación.

Ministerio-Educación, E. (2017). Acuerdo Ministerial Mineduc-2017-00091.

Ortiz, M., Fabara, E. Villagómez, M. e Hidalgo, L. (2017): "La formación y el trabajo docente en el Ecuador", Red Latinoamericana de Estudios sobre Trabajo Docente, constitución en el Ecuador, p. 26

Reyes, L. (2017). Investigación para la formación de profesores. Santiago, Chile: Ediciones UCSH.

Rodríguez, M. C., Hinojosa, L. M. M., \& Ramírez, M. T. G. (2014). Evaluación del desempeño docente, estrés y burnout en profesores universitarios. Rev. Actual Investigación Educativa, 14(1), 1-22.

Román, M. y Murillo, F.J. (2008). "La evaluación del desempeño docente: objeto de disputa y fuente de oportunidades en el campo educativo". Revista Iberoamericana de Evaluación Educativa, 1, 2, 1- 6.

Secretaría de Educación Pública, México. (2012). Programa de Mejoramiento al Profesorado. Recuperado el 12 de abril de 2013 de http://promep.sep.gob.mx/.

Toranzos, L. (2016) En la búsqueda de estándares de calidad. Washington D.C., USA: OEA. 


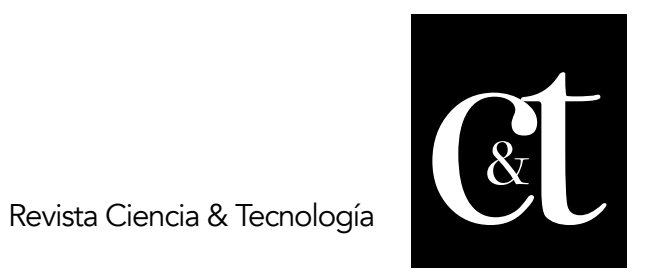

No. 22, 30 de abril de 2019

ISSN impreso: 1390 - 6321

ISSN online: 2661 - 6734

UTPL. (2013). EVA UTPL. Recuperado el 2014, de http://rsa.utpl.edu.ec/material/CCBB/Que\%20son20\%los $20 \%$ estandares de calidad.htm.

Urbay Rodríguez, Marilin. (2006): Modelo de evaluación del desempeño profesional docente como vía para el desarrollo profesional. Recuperado

en

https://dspace.uclv.edu.cu/handle/123456789/8191?show=full

Vaillant, D. (2016). Algunos marcos referenciales en la evaluación del desempeño docente. Revista Iberoamericana de Evaluación Educativa, 1(2). 